\title{
Uterine prolapse and treatment seeking behaviour among women
}

\author{
Ramesh Adhikari ${ }^{*}$ and Ranju $\mathrm{KC}^{2}$ \\ ${ }^{1}$ Mahendra Ratna Campus, Tribhuvan University, Kathmandu, Nepal \\ ${ }^{2}$ School of Planning, Monitoring, Evaluation and Research (SPMER), Kathmandu, Nepal
}

\begin{abstract}
Background: Uterine Prolapse (UP) is one of the emerging public health issues, especially in low income countries like Nepal. Very little is known about UP and the care seeking practices of women with UP in Nepal. This study examines the factors that influence the experience of uterine prolapse and the care seeking behaviour among women in Nepal.

Methods: The data for this paper is extracted from the Nepal Demographic and Health Survey, 2011. We confined the analysis to women who had one or more pregnancy $(n=9,021)$. Bivariate analysis was used to explore the association between the variables and multivariate logistic analysis was used to identify significant predictors of the likelihood of women experiencing uterine prolapse and their care seeking behavior, after controlling for other socio-demographic variables.

Results: More than half of the women were illiterate and almost a fourth of the women (24\%) had no autonomy for their own health care and decision making in terms of household purchasing. More than one out of twenty women (6\%) had experienced symptom(s) of uterine prolapse. It is discouraging to note that more than a third (36\%) who had experienced uterine prolapse did not seek medical care while almost a tenth (9\%) sought traditional treatment. After controlling for socio-demographic variables, our study found that poor women were more likely to experience uterine prolapse $(\mathrm{aOR}=1.16, \mathrm{p}<0.05)$ and also more likely not to seek treatment for uterine prolapse $(\mathrm{aOR}=1.65, \mathrm{p}<0.01)$.

Conclusions: Our study provides novel evidence on an issue of special importance to countries where women suffer uterine prolapse, an issue that has received little attention. A significant proportion of poor women had uterine prolapse. However, care seeking behavior is very low among poor women. Thus, in order to decrease the burden of uterine prolapse as well as to maintain and enhance the well-being of families, programs should focus on alleviating the problem and aim to increase utilization of health care-seeking behavior among women especially among poor women.
\end{abstract}

\section{Background}

Uterine Prolapse (UP) is one of the emerging public health issues, especially in low income countries like Nepal. UP is a condition that occurs when the muscles and ligaments within the pelvis become weak or fragile and are unable to adequately support the uterus [1]. UP often coexists with prolapse of the vaginal walls, involving the bladder or rectum [2]. UP increases discomfort and pain and affects basic day to day activities. As a consequence of various physical problems, the quality of life gets affected and an associated psychosocial problem make these women socially withdrawn as well as affects their ability to work and earn [3]. More than 1 million women suffer from UP in Nepal and most of them belong to the age of 15 to 49 i.e. reproductive age group [4]. A study found that the mean prevalence of uterine prolapse in low- and middle-income countries was $19.7 \%$ [5].

Studies found that poor women generally lack access to health services so they utilize less than the rich women. Few known risk factors for uterine prolapse include extensive physical labor during pregnancy and immediately after delivery, lack of skilled birth attendants during delivery, and smoking [6]. Prolonged labour during childbirth, forced delivery by untrained persons, lack of rest during postpartum period, insufficient spacing between births and poor nutrition are other known risk factors for uterine prolapse [6]. Shakya [7] argues that the problem of UP leads to gender based violence and vice versa.
Studies conducted in different districts/ecological zones of Nepal reported the prevalence of UP is high. UP prevalence varies in different ecological zones, from $20 \%-37 \%$ in the Terai area [8] and $25 \%$ in the far west hills [9] to $27.4 \%$ in the central and eastern hills [10]. Nationally, the prevalence of UP is 7\%-10\% [11-13]. Despite its high prevalence in Nepal, it has not received sufficient attention in Nepal.

Progress in the health sector has been encouraging in Nepal [14]. Government of Nepal has initiated nationwide program to identify and provide necessary treatment and counsel service to women who are suffering from UP. Government of Nepal has started providing free treatment to women suffering from Uterine Prolapse however substantial proportion of women did not seek medical treatment [12]. There is a knowledge gap in literatures in experience of uterine prolapse and treatment seeking behaviour among married women in Nepal. We hope the information regarding factors affecting UP and treatment seeking behaviors are important to guide reproductive health program planners and policy makers and can assist in implementation of reproductive health programs that will decrease UP cases and its complication and increase utilization of the services.

${ }^{\star}$ Correspondence to: Ramesh Adhikari, Mahendra Ratna Campus, Tribhuvan University, Kathmandu, Nepal, E-mail: rameshipsr@gmail.com

Received: April 30, 2018; Accepted: May 29, 2018; Published: June 01, 2018 


\section{Methods}

Data for this paper were drawn from the Nepal Demographic and Health Survey, 2011. The primary purpose of the 2011 NDHS, a nationally representative sample survey, was to provide current and reliable data on fertility and family planning, child mortality, children's nutritional status, utilization of maternal and child health services, domestic violence, and knowledge of HIV/AIDS. The 2011 NDHS was carried out under the aegis of the Population Division of the Ministry of Health and Population. The study protocol was approved by the Nepal Health Research Council and the ICF Macro Institutional Review Board in Calverton, Maryland, USA. All respondents had provided verbal informed consent prior to data collection. Therefore, an independent ethical approval was not required. For this study, we used publicly available dataset from the measure DHS website [2012].

Interviews were conducted among 12,674 women of reproductive age [2012]. However, this analysis is confined to women who had one or more pregnancy $(n=9021)$ to examine the factors affecting uterine prolapse and to women who had uterine prolapse $(n=543)$ to examine the treatment seeking behaviour.

Association between exploratory variables and dependent variables (prevalence of uterine prolapse and treatment seeking behavior) was assessed via bivariate analysis using chi-square tests. Then logistic regression was used to assess the net effect of each exploratory variable on prevalence of uterine prolapse and care seeking behavior after controlling for several other independent variables. Two models were used in the analysis of logistic regression. The first model contained the variables related to wealth. In the second model, other sociodemographic characteristics were added. Prior to the multivariate analysis, multi-collinearity between the variables was assessed. However, no multi-collinearity was found among the variables. Only those variables that were significant in the bivariate analysis were further analyzed in logistic regression. The Statistical Package for Social Science (SPSS 20.0 for Windows) software was used to analyze the data.

\section{Results}

\section{Socio-demographic and economic characteristics}

A fifth of the women (20\%) who had one or more pregnancy were youth aged 15-24. Almost two in five women (38\%) were from Janajati followed by Brahmin/Chhetri ethnic group. More than half of the women had no education (51\%) and almost a third had four or more children ever born (31\%). An overwhelming majority of women were Hindu followed by Buddhist (8\%). More than a fifth of the women did not work $(22 \%)$ while almost half of women were working but not paid (47\%). 29 percent women lived in female headed household. Almost one in five women (18\%) smoked and almost a fourth of the women had no autonomy in household decision (Table 1).

\section{Prevalence of uterine prolapse and treatment seeking behaviour}

Out of 9,021 respondents 6 percent had symptoms of uterine prolapse (Figure 1). Among them, more than a third (36\%) did not seek medical care for prolapse (Figure 2).

\section{Associations with experience of unterine prolapse}

Bivariate analysis shows that wealth, age, ethnicity, education level of women number of children born, religion, ecological zone, place of residence, type of earning and smoking had significant association with experience of uterine prolapse. It is found that 5 percent of rich
Table 1: Background characteristics of women who had one or more pregnancy

\begin{tabular}{|c|c|c|}
\hline Background Characteristics & $\%$ & $\mathbf{N}$ \\
\hline \multicolumn{3}{|l|}{ Age group } \\
\hline Less than 25 years & 20.1 & 1814 \\
\hline $25-34$ & 39.1 & 3525 \\
\hline 35 or above & 40.8 & 3682 \\
\hline \multicolumn{3}{|l|}{ Ethnicity } \\
\hline Brahmin/Chhetri & 34.8 & 3137 \\
\hline Janajati & 38.2 & 3443 \\
\hline Dalit & 14.6 & 1316 \\
\hline Other & 12.5 & 1125 \\
\hline \multicolumn{3}{|l|}{ Education level of women } \\
\hline No education & 51.0 & 4599 \\
\hline Primary & 19.1 & 1721 \\
\hline Secondary or above & 30.0 & 2702 \\
\hline \multicolumn{3}{|l|}{ Total children ever born } \\
\hline None & 1.3 & 118 \\
\hline One & 18.9 & 1704 \\
\hline Two & 27.7 & 2503 \\
\hline Three & 21.4 & 1929 \\
\hline Four or more & 30.7 & 2767 \\
\hline \multicolumn{3}{|l|}{ Religion } \\
\hline Hindu & 84.8 & 7650 \\
\hline Buddhist & 8.2 & 741 \\
\hline Muslim & 3.7 & 336 \\
\hline Kirat/Christian & 3.3 & 295 \\
\hline \multicolumn{3}{|l|}{ Ecological zone } \\
\hline Mountain & 6.6 & 594 \\
\hline Hill & 39.6 & 3574 \\
\hline Terai & 53.8 & 4854 \\
\hline \multicolumn{3}{|l|}{ Place of residence } \\
\hline Urban & 13.2 & 1190 \\
\hline Rural & 86.8 & 7831 \\
\hline \multicolumn{3}{|l|}{ Type of earning from respondent's work } \\
\hline Not working & 21.8 & 1965 \\
\hline Not paid but working & 46.8 & 4225 \\
\hline Paid in Cash or in-kind & 31.4 & 2831 \\
\hline \multicolumn{3}{|l|}{ Wealth index } \\
\hline Poor & 37.0 & 3342 \\
\hline Middle & 20.9 & 1882 \\
\hline Rich & 42.1 & 3798 \\
\hline \multicolumn{3}{|l|}{ Sex of household head } \\
\hline Male & 71.2 & 6425 \\
\hline Female & 28.8 & 2596 \\
\hline \multicolumn{3}{|l|}{ Smoking } \\
\hline Not smoke & 82.1 & 7410 \\
\hline Smoke & 17.9 & 1611 \\
\hline \multicolumn{3}{|l|}{ Women's autonomy in household decision } \\
\hline No autonomy & 23.5 & 2122 \\
\hline Moderate autonomy (involved in 1-2 issues) & 29.8 & 2687 \\
\hline High autonomy (involved in all 3 issues) & 46.7 & 4211 \\
\hline Total & 100.0 & 9021 \\
\hline
\end{tabular}

women had experienced uterine prolapse which was 7 percent among poor women. Similarly a significantly higher percentage of women aged 35 or above (10\%) compared with youth aged less than 25 years $(2 \%)$ had uterine prolapse. Similarly, a significantly higher percentage of Brahmin/Chhetri women (8.2\%), women who had no education (7\%), who had four or more children (8\%), who lived in hill region $(7.6 \%)$, working but not paid (7.1\%) and those who smoked (9.9\%) had uterine prolapse than their counterparts (Table 2). 


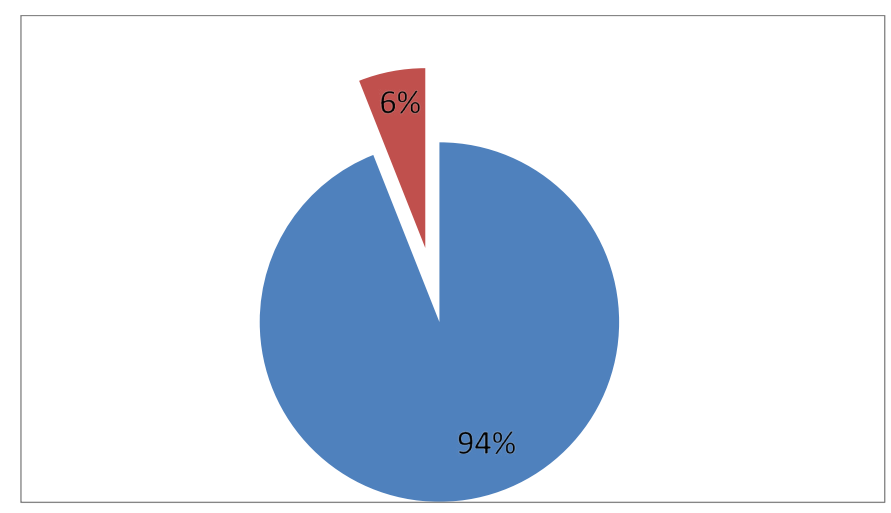

Figure 1: Symptoms of Uterine Prolapse among women who have at least one birth $(\mathrm{n}=9021)$

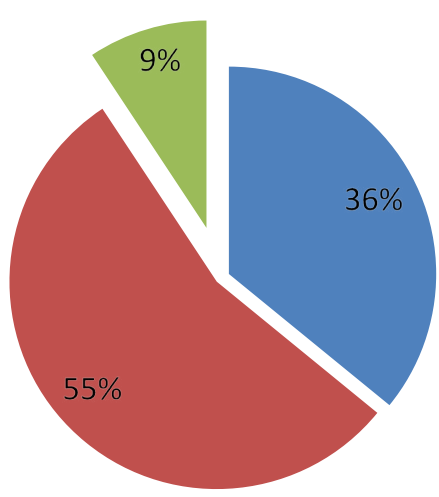

Figure 2: Sought medical treatment for the Uterine Prolapse $(\mathrm{n}=543)$

In the first model of logistic regression, wealth of women had a statistically significant effect on experience of uterine prolapse. Poor women had 38 percent higher odds $(\mathrm{OR}=1.38)$ of experiencing uterine prolapse than rich women. Model 2 presents the final results after adding other socio-demographic characteristics of the women. Even after inclusion of these variables in model 2, wealth retained their significance level. Furthermore, the reduction of the significance level of wealth after inclusion of the other variables indicates that socio-demographic variables are also important predictors for uterine prolapse. Moreover, women's age, ethnicity, religion, and smoking were also significant predictors of experiencing uterine prolapse. Age has a positive and statistically significant impact on experiencing uterine prolapse; as women aged 35 or above were more likely to experience uterine prolapse $(\mathrm{aOR}=3.22)$ than women aged 25 or below. Janajati women were less likely to experience uterine prolapse $(\mathrm{aOR}=0.68)$ than women from Brahmin/Chhetri. In regards to religion, Buddhist women were less likely to experience uterine prolapse $(\mathrm{aOR}=0.53)$ than Hindu women. On the other hand, Muslim women were about 7 times more likely $(\mathrm{aOR}=6.8)$ to experience uterine prolapse than Hindu women. Women who smoked were more likely to experience uterine prolapse $(\mathrm{aOR}=1.37)$ than women who did not smoke (Table 3).

\section{Treatment seeking behaviour}

Among 543 women who had experienced uterine prolapse, 36 percent women did not seek medical treatment. Bivariate analysis shows that wealth, age, ethnicity, religion had statistical significant association with treatment seeking behavior. A significantly higher percentage of women who were from poor family (44\%) than from rich (31\%) and middle class $(28 \%)$ did not seek treatment. A higher percentage of women aged 25-34 (46\%), Dalit (41\%), and Muslim women (73\%) did not seek medical treatment than their counterparts (Table 4).

Wealth index is included in first model of logistic regression. It is found that poor women were more likely $(\mathrm{OR}=1.78)$ not to seek medical

Table 2: Background characteristics of women by experienced signs of uterine prolapse

\begin{tabular}{|c|c|c|c|c|}
\hline Background Characteristics & $\begin{array}{c}\text { Symptoms } \\
\text { of Uterine } \\
\text { Prolapse }\end{array}$ & $\begin{array}{c}\text { No } \\
\text { Symptoms }\end{array}$ & $\%$ & $\mathbf{N}$ \\
\hline \multicolumn{5}{|l|}{ Wealth index ** } \\
\hline Rich & 5.2 & 94.8 & 100.0 & 3798 \\
\hline Middle & 6.0 & 94.0 & 100.0 & 1882 \\
\hline Poor & 7.0 & 93.0 & 100.0 & 3342 \\
\hline \multicolumn{5}{|l|}{ Age group *** } \\
\hline Less than 25 years & 2.2 & 97.8 & 100.0 & 1814 \\
\hline $25-34$ & 4.1 & 95.9 & 100.0 & 3525 \\
\hline 35 or above & 9.7 & 90.3 & 100.0 & 3682 \\
\hline \multicolumn{5}{|l|}{ Ethnicity *** } \\
\hline Brahmin/Chhetri & 8.2 & 91.8 & 100.0 & 3137 \\
\hline Janajati & 5.2 & 94.8 & 100.0 & 3443 \\
\hline Dalit & 6.8 & 93.2 & 100.0 & 1316 \\
\hline Other & 1.5 & 98.5 & 100.0 & 1125 \\
\hline \multicolumn{5}{|l|}{ Education level of women $* * *$} \\
\hline No education & 7.0 & 93.0 & 100.0 & 4599 \\
\hline Primary & 6.3 & 93.7 & 100.0 & 1721 \\
\hline Secondary or above & 4.2 & 95.8 & 100.0 & 2702 \\
\hline \multicolumn{5}{|l|}{ Total children born*** } \\
\hline None & 3.9 & 96.1 & 100.0 & 118 \\
\hline One & 1.9 & 98.1 & 100.0 & 1704 \\
\hline Two & 5.7 & 94.3 & 100.0 & 2503 \\
\hline Three & 7.3 & 92.7 & 100.0 & 1929 \\
\hline Four or more & 8.0 & 92.0 & 100.0 & 2767 \\
\hline \multicolumn{5}{|l|}{ Religion ** } \\
\hline Hindu & 6.3 & 93.7 & 100.0 & 7650 \\
\hline Buddhist & 3.5 & 96.5 & 100.0 & 741 \\
\hline Muslim & 4.0 & 96.0 & 100.0 & 336 \\
\hline Kirat/Christian & 6.2 & 93.8 & 100.0 & 295 \\
\hline \multicolumn{5}{|l|}{ Ecological zone $* * *$} \\
\hline Mountain & 7.3 & 92.7 & 100.0 & 594 \\
\hline Hill & 7.6 & 92.4 & 100.0 & 3574 \\
\hline Terai & 4.7 & 95.3 & 100.0 & 4854 \\
\hline \multicolumn{5}{|l|}{ Place of residence * } \\
\hline Urban & 4.6 & 95.4 & 100.0 & 1190 \\
\hline Rural & 6.2 & 93.8 & 100.0 & 7831 \\
\hline \multicolumn{5}{|c|}{ Type of earning from respondent's work $* * *$} \\
\hline Not working & 4.4 & 95.6 & 100.0 & 1965 \\
\hline Not paid but working & 7.1 & 92.9 & 100.0 & 4225 \\
\hline paid in Cash or in-kind & 5.5 & 94.5 & 100.0 & 2831 \\
\hline \multicolumn{5}{|l|}{ Sex of household head } \\
\hline Male & 6.2 & 93.8 & 100.0 & 6425 \\
\hline Female & 5.7 & 94.3 & 100.0 & 2596 \\
\hline \multicolumn{5}{|l|}{ Smoking *** } \\
\hline Not smoke & 5.2 & 94.8 & 100.0 & 7410 \\
\hline Smoke & 9.9 & 90.1 & 100.0 & 1611 \\
\hline \multicolumn{5}{|c|}{ Women's autonomy in household decision } \\
\hline No autonomy & 5.1 & 94.9 & 100.0 & 2122 \\
\hline $\begin{array}{l}\text { Moderate autonomy (involved } \\
\text { in 1-2 issues) }\end{array}$ & 6.6 & 93.4 & 100.0 & 2687 \\
\hline $\begin{array}{l}\text { High autonomy (involved in } \\
\text { all } 3 \text { issues) }\end{array}$ & 6.1 & 93.9 & 100.0 & 4211 \\
\hline Total & 6.0 & 94.0 & 100.0 & 9021 \\
\hline
\end{tabular}


Table 3: Adjusted odds ratios (aOR) from multivariable logistic regression assessing the likelihood of experiencing uterine prolapse by women's wealth and selected social and demographic characteristics

\begin{tabular}{|c|c|c|c|c|c|c|}
\hline \multirow{3}{*}{ Predicators } & \multicolumn{3}{|c|}{ Model I } & \multicolumn{3}{|c|}{ Model II } \\
\hline & \multirow[b]{2}{*}{ OR } & \multicolumn{2}{|c|}{$95 \% \mathrm{CI}$} & \multirow[b]{2}{*}{ aOR } & \multicolumn{2}{|c|}{$95 \%$ CI } \\
\hline & & Lower & Upper & & Lower & Upper \\
\hline \multicolumn{7}{|l|}{ Wealth index } \\
\hline Rich (ref.) & 1.00 & & & 1.00 & & \\
\hline Middle & 1.17 & .92 & 1.49 & 1.11 & .86 & 1.46 \\
\hline Poor & $1.38 * *$ & 1.14 & 1.68 & $1.16^{*}$ & 1.09 & 1.49 \\
\hline \multicolumn{7}{|l|}{ Age group } \\
\hline Less than 25 years (ref.) & & & & 1.00 & & \\
\hline $25-34$ & & & & 1.42 & 0.96 & 2.09 \\
\hline 35 or above & & & & $3.22 * * *$ & 2.15 & 4.81 \\
\hline \multicolumn{7}{|l|}{ Ethnicity } \\
\hline Brahmin/Chhetri (ref.) & & & & 1.00 & & \\
\hline Janajati & & & & $0.68 * *$ & 0.54 & 0.85 \\
\hline Dalit & & & & 0.84 & 0.64 & 1.11 \\
\hline Other & & & & $0.09 * * *$ & 0.04 & 0.21 \\
\hline \multicolumn{7}{|l|}{ Education level of women } \\
\hline No education (ref.) & & & & 1.00 & & \\
\hline Primary & & & & 1.11 & 0.87 & 1.42 \\
\hline Secondary or above & & & & 0.88 & 0.66 & 1.16 \\
\hline \multicolumn{7}{|l|}{ Total children } \\
\hline None (ref.) & & & & 1.00 & & \\
\hline One & & & & 0.49 & 0.18 & 1.32 \\
\hline Two & & & & 1.017 & 0.39 & 2.68 \\
\hline Three & & & & 1.06 & 0.39 & 2.83 \\
\hline Four or more & & & & 0.86 & 0.32 & 2.31 \\
\hline \multicolumn{7}{|l|}{ Religion } \\
\hline Hindu (ref.) & & & & 1.00 & & \\
\hline Buddhist & & & & $0.53^{* *}$ & 0.34 & 0.82 \\
\hline Muslim & & & & $6.81 * * *$ & 2.45 & 18.96 \\
\hline Kirat/Christian & & & & 1.05 & 0.64 & 1.73 \\
\hline \multicolumn{7}{|l|}{ Ecological zone } \\
\hline Mountain (ref.) & & & & 1.00 & & \\
\hline Hill & & & & 1.22 & 0.86 & 1.72 \\
\hline Terai & & & & 0.91 & 0.63 & 1.32 \\
\hline \multicolumn{7}{|l|}{ Place of residence } \\
\hline Urban (ref.) & & & & 1.00 & & \\
\hline Rural & & & & 1.23 & 0.90 & 1.68 \\
\hline \multicolumn{7}{|c|}{ Type of earning from respondent's work } \\
\hline Not working (ref.) & & & & 1.00 & & \\
\hline Not paid but working & & & & 0.92 & 0.69 & 1.23 \\
\hline paid in Cash or in-kind & & & & 0.84 & 0.63 & 1.12 \\
\hline \multicolumn{7}{|l|}{ Smoking } \\
\hline Not smoke (ref.) & & & & 1.00 & & \\
\hline Smoke & & & & $1.37 * *$ & 1.10 & 1.69 \\
\hline Constant & \multicolumn{3}{|c|}{$0.055^{* * *}$} & \multicolumn{3}{|c|}{$0.036^{* * *}$} \\
\hline Cox \& Snell R Square & \multicolumn{3}{|c|}{0.001} & \multicolumn{3}{|c|}{0.034} \\
\hline-2 Log likelihood & \multicolumn{3}{|c|}{4095.8} & & 3795.5 & \\
\hline
\end{tabular}

treatment of uterine prolapse than rich women. Model 2 presents the final results after adding other socio-demographic characteristics of the women. Even after inclusion of these variables in model 2, wealth retained the significance level. Poor women were more likely not to seek treatment $(\mathrm{aOR}=1.65)$ for the uterine prolapse after controlling for other variables (Table 5).

\section{Discussion}

This study has attempted to investigate the influence of particular socio-demographic, economic, and cultural factors-especially wealth on experience of UP and care seeking behaviour. The present study shows that the experience of uterine prolapse and lack of treatment seeking behavior is common among Nepalese women and this indicates an unmet need for an effective reproductive health program.

The bivariate analysis shows that variables such as wealth, age, ethnicity, education level of women, total number of children born, religion, ecological zone, place of residence, type of earning from

Table 4: Background characteristics of Women by treatment seeking behaviour of signs of uterine prolapsed

\begin{tabular}{|c|c|c|c|c|}
\hline \multirow[t]{2}{*}{ Background Characteristics } & \multicolumn{2}{|c|}{$\begin{array}{c}\text { Treatment seeking behaviour } \\
\text { of uterine prolapse }\end{array}$} & \multicolumn{2}{|c|}{ Total } \\
\hline & Not treated & Treated & $\%$ & $\mathbf{N}$ \\
\hline \multicolumn{5}{|l|}{ Wealth index** } \\
\hline Rich & 30.7 & 69.3 & 100.0 & 196 \\
\hline Middle & 28.3 & 71.7 & 100.0 & 113 \\
\hline Poor & 44.1 & 55.9 & 100.0 & 234 \\
\hline \multicolumn{5}{|l|}{ Age group ** } \\
\hline Less than 25 years & 38.5 & 61.5 & 100.0 & 40 \\
\hline $25-34$ & 45.9 & 54.1 & 100.0 & 145 \\
\hline 35 or above & 31.6 & 68.4 & 100.0 & 358 \\
\hline \multicolumn{5}{|l|}{ Ethnicity* } \\
\hline Brahmin/Chhetri & 33.8 & 66.2 & 100.0 & 258 \\
\hline Janajati & 33.9 & 66.1 & 100.0 & 178 \\
\hline Dalit & 40.5 & 59.5 & 100.0 & 89 \\
\hline Other & 64.7 & 35.3 & 100.0 & 17 \\
\hline \multicolumn{5}{|l|}{ Education level of women } \\
\hline No education & 36.8 & 63.2 & 100.0 & 323 \\
\hline Primary & 30.9 & 69.1 & 100.0 & 108 \\
\hline Secondary or above & 38.3 & 61.7 & 100.0 & 112 \\
\hline \multicolumn{5}{|l|}{ Total children } \\
\hline None & & 100.0 & 100.0 & 5 \\
\hline One & 32.3 & 67.7 & 100.0 & 33 \\
\hline Two & 36.1 & 63.9 & 100.0 & 143 \\
\hline Three & 35.2 & 64.8 & 100.0 & 141 \\
\hline Four or more & 37.6 & 62.4 & 100.0 & 221 \\
\hline \multicolumn{5}{|l|}{ Religion* } \\
\hline Hindu & 35.8 & 64.2 & 100.0 & 486 \\
\hline Buddhist & 24.6 & 75.4 & 100.0 & 26 \\
\hline Muslim & 72.9 & 27.1 & 100.0 & 14 \\
\hline Kirat/Christian & 28.6 & 71.4 & 100.0 & 18 \\
\hline \multicolumn{5}{|l|}{ Ecological zone } \\
\hline Mountain & 34.7 & 65.3 & 100.0 & 43 \\
\hline Hill & 38.8 & 61.2 & 100.0 & 273 \\
\hline Terai & 32.8 & 67.2 & 100.0 & 227 \\
\hline \multicolumn{5}{|l|}{ Place of residence } \\
\hline Urban & 26.5 & 73.5 & 100.0 & 55 \\
\hline Rural & 37.0 & 63.0 & 100.0 & 488 \\
\hline \multicolumn{5}{|c|}{ Type of earning from respondent's work } \\
\hline Not working & 33.5 & 66.5 & 100.0 & 86 \\
\hline Not paid but working & 39.4 & 60.6 & 100.0 & 301 \\
\hline paid in Cash or in-kind & 30.7 & 69.3 & 100.0 & 156 \\
\hline \multicolumn{5}{|l|}{ Sex of household head } \\
\hline Male & 36.3 & 63.7 & 100.0 & 397 \\
\hline Female & 35.0 & 65.0 & 100.0 & 147 \\
\hline \multicolumn{5}{|c|}{ Women's autonomy in household decision } \\
\hline No autonomy & 40.5 & 59.5 & 100.0 & 108 \\
\hline $\begin{array}{l}\text { Moderate autonomy (involved in } \\
1-2 \text { issues) }\end{array}$ & 37.9 & 62.1 & 100.0 & 176 \\
\hline $\begin{array}{l}\text { High autonomy (involved in all } 3 \\
\text { issues) }\end{array}$ & 32.7 & 67.3 & 100.0 & 259 \\
\hline Total & 35.9 & 64.1 & 100.0 & 543 \\
\hline
\end{tabular}

Note $* *$ Significant at $\mathrm{p}<0.001 ; * *=\mathrm{p}<0.01$ and $*=\mathrm{p}<0.05$ 
Table 5: Adjusted odds ratios (aOR) from multivariable logistic regression assessing the likelihood of not seeking medical treatment by women's wealth and selected social and demographic characteristics.

\begin{tabular}{|c|c|c|c|c|c|c|}
\hline \multirow{3}{*}{ Predicators } & \multicolumn{3}{|c|}{ Model I } & \multicolumn{3}{|c|}{ Model II } \\
\hline & \multirow{2}{*}{ OR } & \multicolumn{2}{|c|}{$95 \% \mathrm{CI}$} & \multirow{2}{*}{ aOR } & \multicolumn{2}{|c|}{$95 \% \mathrm{CI}$} \\
\hline & & Lower & Higher & & Lower & Higher \\
\hline \multicolumn{7}{|l|}{ Wealth index } \\
\hline Rich (ref.) & 1.00 & & & 1.00 & & \\
\hline Middle & 0.89 & 0.54 & 1.48 & 0.77 & 0.44 & 1.33 \\
\hline Poor & $1.78 * *$ & 1.19 & 2.65 & $1.65^{* *}$ & 1.08 & 2.52 \\
\hline \multicolumn{7}{|l|}{ Age group } \\
\hline Less than 25 years (ref.) & & & & 1.00 & & \\
\hline $25-34$ & & & & 1.19 & 0.57 & 2.50 \\
\hline 35 or above & & & & 0.77 & 0.38 & 1.54 \\
\hline \multicolumn{7}{|l|}{ Ethnicity } \\
\hline Brahmin/Chhetri (ref.) & & & & 1.00 & & \\
\hline Janajati & & & & 0.93 & 0.59 & 1.47 \\
\hline Dalit & & & & 1.17 & 0.69 & 1.98 \\
\hline Other & & & & 1.19 & 0.14 & 9.91 \\
\hline \multicolumn{7}{|l|}{ Religion } \\
\hline Hindu (ref.) & & & & 1.00 & & \\
\hline Buddhist & & & & 0.58 & 0.22 & 1.54 \\
\hline Muslim & & & & 4.47 & 0.39 & 51.15 \\
\hline Kirat/Christian & & & & 0.84 & 0.28 & 2.49 \\
\hline Constant & \multicolumn{3}{|c|}{0.79} & \multicolumn{3}{|c|}{0.86} \\
\hline-2 Log likelihood & \multicolumn{3}{|c|}{697.7} & \multicolumn{3}{|c|}{681.1} \\
\hline Cox \& Snell R Square & \multicolumn{3}{|c|}{0.022} & \multicolumn{3}{|c|}{0.051} \\
\hline
\end{tabular}

Note $* * *$ Significant at $\mathrm{p}<0.001 ; * *=\mathrm{p}<0.01$ and $*=\mathrm{p}<0.05$

respondent's work, and smoking are important in explaining uterine prolapse. The multivariate analysis supports many of the findings of the bivariate analysis. In the multivariate analysis, wealth, age, ethnicity, religion and smoking are found to have a statistically significant influence on experience of uterine prolapse. In regards to treatment seeking behavior, bivariate analysis shows that wealth, age, ethnicity and religion have significant association with treatment seeking behaviour of uterine prolapse. Multivariate analysis shows that wealth is significant predicators for treatment seeking behaviour after controlling other socio-demographic variables.

Wealth status seems to be strong predicators among many other variables for both experiencing uterine prolapse and care seeking behaviour. Poor women were more likely to experience uterine prolapse. However these poor women were also more likely not to seek treatment for the problem. Although government of Nepal provides free treatment, poor women may not be able to manage travel and other additional cost that incur during the treatment. The other reason can be poor women generally lack access to health services.

This study has some potential limitations that must be understood in the light of the results. Due to the cross-sectional design of the study and all of the items analyzed came from information at the time of survey, so the analysis cannot show cause and effect relationship.

\section{Conclusions}

Our study provides novel evidence on an issue of special importance to countries where women suffer uterine prolapse, an issue that has received little attention in the country. A significant proportion of women especially poor women had uterine prolapse. However care seeking behavior is very low among these poor women. Thus, in order to decrease uterine prolapse among women as well as to maintain and enhance the well-being of families, programs should focus on alleviating the uterine prolapse and aim to increase utilization of health care-seeking behavior among women especially among poor women.

\section{Acknowledgment} data.

The author thanks MEASURE DHS + for providing access to the

\section{Availability of data and material}

The data used are publicly available from the MEASURE DHS site.

\section{Authors' contributions}

RA conducted data analysis, interpretation, and drafted the manuscript. RKC was involved in the interpretation of the data. All authors read and approved the final manuscript.

\section{Ethics approval and consent to participate}

The study protocol was approved by the Nepal Health Research Council and the ICF Macro Institutional Review Board in Calverton, Maryland, USA. All respondents had provided verbal informed consent to be interviewed prior to data collection. Therefore, an independent ethical approval was not required. For this study, we used publicly available dataset from the measure DHS website.

\section{References}

1. Lori Smith BSN MSN CRNP (2016) Uterine Prolapse: Causes, Symptoms and Treatment. Medical News Today.

2. Doshani A, Teo REC, Mayne CJ, Tincello DJ (2007) Clinical Review-Uterine Prolapse. BMJ 335: 819-823. [Crossref]

3. Ravindra STK, Savitri R, Bhavani A (2017) Women's experiences of utero vaginal prolapse: a qualitative study from Tamil Nadu, India.

4. Bodner-Adler B, Shrivastava C, Bodner K (2007) Risk factors for uterine prolapse in Nepal. J Int Urogynecol J Pelvic Floor Dysfunct 18: 1343-1346. [Crossref]

5. Godfrey, Walker JA, Gunasekera P (2011) Pelvic organ prolapse and incontinence in developing countries: review of prevalence and risk factors. Int Urogynecol $J$ 22: $127-$ 135. [Crossref]

6. Upreti A, Bhattarai K, Onta SR (2001) Where there is no doctor, RHEST-Nepal, PHECT-Nepal.

7. Shakya T (2006) Uterus Prolapse Leading to Gender Based Violence, RHIYA/PHECTNepal

8. Pradhan S (2007) Unheeded Agonies: A Study of Uterine Prolapse Prevalence and Its Causes in Siraha and Saptari Districts, Nepal. Centre for Agro-Ecology and Development Kathmandu.

9. Bonetti TR, Erpelding A, Pathak LR (2004) Listening to "felt needs": investigating genital prolapse in Western Nepal. Reprod Health Matters 12: 166-175. [Crossref]

10. Messerchmidt L (2009) Uterine prolapse in Nepal: the rural health developmen Project's response. JNEPHA 4: 33-42.

11. Ministry of Health and Population (MoHP) [Nepal] (2007) New Era and ICF International Inc. Nepal demographic and health survey 2006. Calverton: MoHP, New Era and ICF International Inc.

12. Ministry of Health and Population (MoHP) [Nepal] (2012) New Era and ICF International Inc. Nepal demographic and health survey 2011. Calverton: MoHP, New Era and ICF International Inc.

13. Gurung G, Rana A, Amatya A, Bishta KD, Joshi AB, et al. (2007) Pelvic organ prolapse in rural Nepalese women of reproductive age groups: what makes it so common. $\mathrm{NJ}$ Obstet Gynaecol 2: 35-41.

14. NPC (2015) Sustainable Development Goals 2016-2030. National (Preliminary) Report. Government of Nepal, National Planning Commission.

Copyright: (C)2018 Adhikari R. This is an open-access article distributed under the terms of the Creative Commons Attribution License, which permits unrestricted use, distribution, and reproduction in any medium, provided the original author and source are credited. 